\title{
The Hubbard model: bosonic excitations and zero-frequency constants
}

\author{
Adolfo Avella* Ferdinando Mancini \\ Dipartimento di Fisica "E.R. Caianiello" - Unità INFM di Salerno \\ Università degli Studi di Salerno, I-84081 Baronissi (SA), Italy
}

\begin{abstract}
A fully self-consistent calculation of the bosonic dynamics of the Hubbard model is developed within the Composite Operator Method. From one side we consider a basic set of fermionic composite operators (Hubbard fields) and calculate the retarded propagators. On the other side we consider a basic set of bosonic composite operators (charge, spin and pair) and calculate the causal propagators. The equations for the Green's functions (GF) (retarded and causal), studied in the polar approximation, are coupled and depend on a set of parameters not determined by the dynamics. First, the pair sector is self-consistently solved together with the fermionic one and the zero-frequency constants (ZFC) are calculated not assuming the ergodic value, but fixing the representation of the GF in such a way to maintain the constrains required by the algebra of the composite fields. Then, the scheme to compute the charge and spin sectors, ZFCs included, is given in terms of the fermionic and pair correlators.
\end{abstract}

Key words: Hubbard model, Composite Operator Method, bosonic excitations, zero-frequency constants $P A C S$ :

Recently, the Green's function method for composite operators has been revisited [1]. In particular, it has been shown that the formulation generates an internal self-consistency which cannot be solved uniquely by the dynamics, but ingredients related to the microscopic nature of the local operator algebra and to the macroscopic nature of the external boundary conditions must be provided. This is not surprising. The properties of composite operators are not known at priori, they have a microscopic nature but manifest at level of observation; as a consequence they are self-consistently deter-

\footnotetext{
* Dipartimento di Fisica "E.R. Caianiello"

Unità di Ricerca INFM di Salerno

Università degli Studi di Salerno

Via S. Allende, I-84081 Baronissi (SA), Italy

Tel. +39089 965418

Fax: +39089 965275

Email addresses: avella@sa.infn.it (Adolfo Avella), mancini@sa.infn.it (Ferdinando Mancini).

URLs: http://scs.sa.infn.it/avella (Adolfo Avella), http://scs.sa.infn.it/mancini (Ferdinando Mancini).
}

mined by the dynamics of the system, by the algebra and by the boundary conditions.

Roughly, the properties of electronic systems can be classified in two large classes: single particle properties, described in terms of fermionic propagators, and response functions, described in terms of bosonic propagators. These two sectors, fermionic and bosonic, are not independent, and a fully self-consistent solution requires that both sectors are simultaneously solved. All the new theoretical schemes, developed in the last years, show the importance of the spin and charge correlations in order to describe the single particle properties of highly correlated electron systems.

In order to illustrate these ideas, we consider the Hubbard model, described by the Hamiltonian

$$
H=\sum_{\mathbf{i}, \mathbf{j}}\left(t_{\mathbf{i} \mathbf{j}}-\mu \delta_{\mathbf{i j}}\right) c^{\dagger}(\mathbf{i}, t) c(\mathbf{j}, t)+U \sum_{\mathbf{i}} n_{\uparrow}(i) n_{\downarrow}(i)
$$

We use the standard notation: $c(i), c^{\dagger}(i)$ are annihilation and creation operators of electrons in the spinor 
notation; $\mathbf{i}$ stays for the lattice vector and $i=(\mathbf{i}, t)$; $\mu$ is the chemical potential; $t_{\mathbf{i j}}$ denotes the transfer integral; $U$ is the screened Coulomb potential; $n_{\sigma}(i)=$ $c_{\sigma}^{\dagger}(i) c_{\sigma}(i)$ is the charge density of electrons at the site i with spin $\sigma$. For a cubic lattice and by considering only nearest neighbor sites the hopping matrix has the form $t_{\mathbf{i j}}=-2 d t \alpha_{\mathbf{i j}}$, where $d$ is the dimension and $\alpha_{i j}$ is the projection operator

$\alpha_{\mathbf{i j}}=\frac{1}{N} \sum_{\mathbf{k}} e^{\mathrm{i} \mathbf{k} \cdot\left(R_{\mathbf{i}}-R_{\mathbf{j}}\right)} \alpha(\mathbf{k}) \quad \alpha(\mathbf{k})=\frac{1}{d} \sum_{n=1}^{d} \cos \left(k_{n}\right)$

We choose as fermionic basis

$$
\psi(i)=\left(\begin{array}{l}
\xi(i) \\
\eta(i)
\end{array}\right)
$$

where $\xi(i)=[1-n(i)] c(i)$ and $\eta(i)=n(i) c(i)$ are the Hubbard operators, and $n(i)=\sum_{\sigma} n_{\sigma}(i)$. In the two-pole approximation [2] the retarded GF $G(i, j)=$ $\left\langle R\left[\psi(i) \psi^{\dagger}(j)\right]\right\rangle$ satisfies the equation

$$
[\omega-\varepsilon(\mathbf{k})] G(k, \omega)=I(\mathbf{k})
$$

where $I(\mathbf{k})=F \cdot T \cdot\left\langle\left\{\psi(\mathbf{i}, t), \psi^{\dagger}(\mathbf{j}, t)\right\}\right\rangle$ and $\varepsilon(\mathbf{k})=$ F.T. $\left\langle\left\{\mathrm{i} \frac{\partial \psi(\mathbf{i}, t)}{\partial t}, \psi^{\dagger}(\mathbf{j}, t)\right\}\right\rangle I^{-1}(\mathbf{k})$; the symbol F.T. denotes the Fourier transform. In the paramagnetic phase the energy matrix $\varepsilon(\mathbf{k})$ depends on the following set of internal parameters: $\mu, \Delta=\left\langle\xi^{\alpha}(i) \xi^{\dagger}(i)\right\rangle-\left\langle\eta^{\alpha}(i) \eta^{\dagger}(i)\right\rangle$, $p=\left\langle n_{\mu}^{\alpha}(i) n_{\mu}(i)\right\rangle / 4-\left\langle\left[c_{\uparrow}(i) c_{\downarrow}(i)\right]^{\alpha} c_{\downarrow}^{\dagger}(i) c_{\uparrow}^{\dagger}(i)\right\rangle$, which must be self-consistently determined. Given an operator $\zeta(i)$, we are using the notation $\zeta^{\alpha}(i)=\sum_{\mathbf{j}} \alpha_{\mathbf{i j}} \zeta(\mathbf{j}, t)$. The operator $n_{\mu}(i)=c^{\dagger}(i) \sigma_{\mu} c(i)\left[\sigma_{\mu}=(\mathbf{1}, \sigma), \sigma\right.$ are the Pauli matrices] is the charge $(\mu=0)$ and spin ( $\mu=1,2,3)$ density operator. The local algebra satisfied by the fermionic field (3) imposes the constraint $\left\langle\xi(i) \eta^{\dagger}(i)\right\rangle=0$ : this equation allows us to solve selfconsistently the fermionic sector. However, it is worth noticing that the presence of the parameter $p$ directly relates the fermionic sector to bosonic sectors.

We consider then the composite bosonic field

$$
N^{(\mu)}(i)=\left(\begin{array}{c}
n_{\mu}(i) \\
\rho_{\mu}(i)
\end{array}\right)
$$

where $\rho_{\mu}(i)=c^{\dagger}(i) \sigma_{\mu} c^{\alpha}(i)-c^{\alpha \dagger}(i) \sigma_{\mu} c(i)$. In the twopole approximation [3] the causal $\mathrm{GF}^{(\mu)}(i, j)=$ $\left\langle T\left[N^{(\mu)}(i) N^{(\mu) \dagger}(j)\right]\right\rangle$ satisfies the equation

$$
\left[\omega-\varepsilon^{(\mu)}(\mathbf{k})\right] G^{(\mu)}(\mathbf{k}, \omega)=I^{(\mu)}(\mathbf{k})
$$

where $I^{(\mu)}(\mathbf{k})=F . T \cdot\left\langle\left[N^{(\mu)}(\mathbf{i}, t), N^{(\mu) \dagger}(\mathbf{j}, t)\right]\right\rangle$ and $\varepsilon^{(\mu)}(\mathbf{k})=F . T .\left\langle\left[\mathrm{i} \frac{\partial N^{(\mu)}(\mathbf{i}, t)}{\partial t}, N^{(\mu) \dagger}(\mathbf{j}, t)\right]\right\rangle\left[I^{(\mu)}(\mathbf{k})\right]^{-1}$. In the one-dimensional case (we consider the $1 \mathrm{D}$ case just for the sake of simplicity) the energy matrix $\varepsilon^{(\mu)}(\mathbf{k})$ depends on the following set of internal parameters: (i) fermionic parameters: $C^{\alpha}=\left\langle c^{\alpha}(i) c^{\dagger}(i)\right\rangle$,

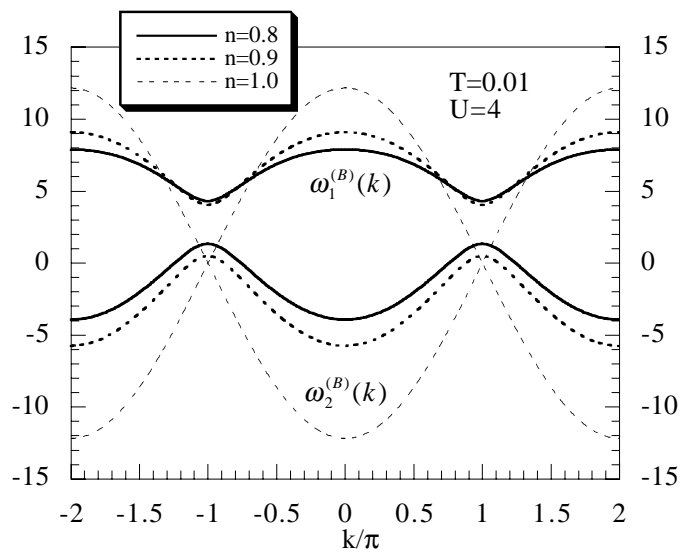

Fig. 1. Pair energy spectra $\omega_{n}^{(B)}(k)$ as a function of the momentum $k$ for Coulomb repulsion $U=4$, temperature $T=0.01$ and filling $n=0.8,0.9$ and 1 .

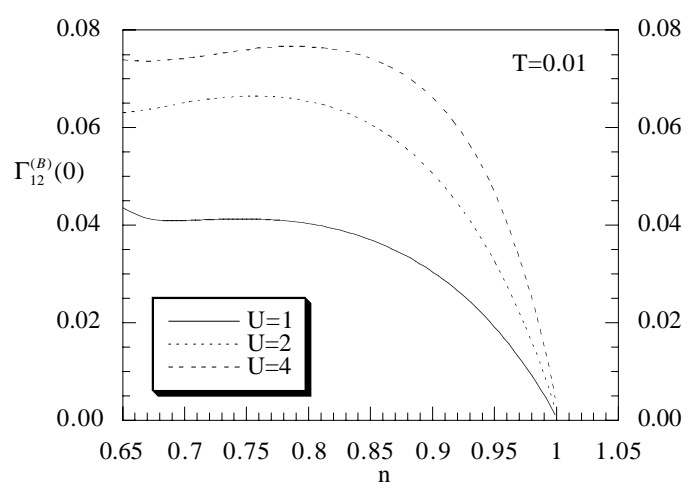

Fig. 2. Pair zero-frequency constant $\Gamma_{12}^{(B)}(\mathbf{0})$ as a function of the filling $n$ for temperature $T=0.01$ and Coulomb repulsion $U=1,2$ and 4 .

$C^{\eta}=\left\langle c^{\eta}(i) c^{\dagger}(i)\right\rangle, C^{\lambda}=\left\langle c^{\lambda}(i) c^{\dagger}(i)\right\rangle, E=\left\langle\eta(i) \eta^{\dagger}(i)\right\rangle$, $E^{\eta}=\left\langle\eta^{\eta}(i) c^{\dagger}(i)\right\rangle$, where $\eta_{i j}, \lambda_{i j}$ are the projection operators on the second and third nearest neighbors, respectively; (ii) bosonic parameters: $a_{\mu}, b_{\mu}$ and $c_{\mu}$, whose explicit expressions, although for the 2D case, are reported in Ref. [3]; (iii) zero-frequency functions (ZFF) $\Gamma^{(\mu)}(\mathbf{i}, \mathbf{j})$ (see Ref. [1]). Due to the hydrodynamic constraints, two bosonic parameters should be determined as $b_{\mu}=a_{\mu}+n-2\left(E-E^{\eta}\right)$ and $c_{\mu}=a_{\mu}-n+2\left(E-E^{\eta}\right)$. The parameter $a_{\mu}$ instead can be determined by means of the local algebra constrain $\left\langle n_{\mu}(i) n_{\mu}(i)\right\rangle=\langle n\rangle+2\left(2 \delta_{\mu, 0}-1\right) D$, where $D=\langle n\rangle / 2-E$ is the double occupancy. The ZFF are left undetermined.

According to this, we need another composite bosonic field

$$
B(i)=\left(\begin{array}{l}
h(i) \\
f(i)
\end{array}\right)
$$

where $h(i)=c_{\uparrow}(i) c_{\downarrow}(i)$ and $f(i)=c_{\uparrow}^{\alpha}(i) c_{\downarrow}(i)+$ $c_{\uparrow}(i) c_{\downarrow}^{\alpha}(i)$. In the two-pole approximation the causal 
GF $G^{(B)}(i, j)=\left\langle T\left[B(i) B^{\dagger}(j)\right]\right\rangle$ satisfies the equation

$$
\left[\omega-\varepsilon^{(B)}(k)\right] G^{(B)}(k, \omega)=I^{(B)}(k)
$$

where $I^{(B)}(k)=F \cdot T \cdot\left\langle\left[B(\mathbf{i}, t), B^{\dagger}(\mathbf{j}, t)\right]\right\rangle$ and $\varepsilon^{(B)}(k)=$ F.T. $\left\langle\left[\mathrm{i} \frac{\partial B(\mathbf{i}, t)}{\partial t}, B^{\dagger}(\mathbf{j}, t)\right]\right\rangle\left[I^{(B)}(k)\right]^{-1}$. The energy matrix $\varepsilon^{(B)}(k)$ depends on the following set of internal parameters: (i) fermionic parameters: $C^{\alpha}, C^{\eta}, C^{\lambda}, E, E^{\eta}$; (ii) bosonic parameters: $u, v$ and $w$, whose lengthy expressions are not reported here for the sake of brevity; (iii) $\mathrm{ZFF} \Gamma^{(B)}(\mathbf{i}, \mathbf{j})$. The condition that the pair energy spectra $\omega_{n}^{(B)}(k)$ are finite and the local algebra constrain $\left\langle h(i) h^{\dagger}(i)\right\rangle=1-\langle n\rangle+D$ completely determine the parameters $u, v$ and $w$. The algebra constraint $\left\langle h(i) f^{\dagger}(i)\right\rangle=2\left\langle\xi^{\alpha}(i) c^{\dagger}(i)\right\rangle$ will be used to compute the ZFC $\Gamma_{12}^{(B)}(\mathbf{0})$. Then, the pair sector can be immediately solved once the solution for the fermionic sector has been found. The results of this scheme are shown in Figs. 1 and 2, where the pair energy spectra and $\Gamma_{12}^{(B)}(\mathbf{0})$ are shown, respectively. It is worth noticing that, in the present scheme, the pair dynamics is ergodic only at half-filling.

Now, once we have solved the fermionic and pair sectors we can come back to the charge-spin one. We need to compute the six ZFC: $\Gamma_{11}^{(0)}(0), \Gamma_{11}^{(0)}(a), \Gamma_{11}^{(3)}(0)$, $\Gamma_{11}^{(3)}(a), \Gamma_{22}^{(0)}(0), \Gamma_{22}^{(3)}(0)$. They can be fixed by means of as many algebra constrains coming from the expressions of the following correlators: $\left\langle n_{\mu}(i) n_{\mu}^{\alpha}(i)\right\rangle$, $\left\langle\rho_{\mu}(i) \rho_{\mu}^{\alpha}(i)\right\rangle,\left\langle h^{\alpha}(i) h^{\dagger}(i)\right\rangle$ and $\left\langle f(i) f^{\dagger}(i)\right\rangle$. The explicit expressions of the constraints are quite lengthy and will be given elsewhere.

In conclusion, we have reported a fully self-consistent scheme of calculations for both the fermionic and the three (spin, charge and pair) bosonic sectors of the Hubbard model. It is worth noticing that, within this scheme, the ZFC of the spin and charge sectors, which could assume, at least in principle, not ergodic values as the pair one does, can be self-consistently computed and give invaluable information regarding the dynamics of the corresponding operators.

\section{References}

[1] F. Mancini, A. Avella, Pauli Principle, Green's Functions and Equations of Motion, cond-mat/0006377 (2000).

[2] A. Avella, F. Mancini, D. Villani, L. Siurakshina, V. Y. Yushankhai, The Hubbard model in the two-pole approximation, Int. J. Mod. Phys. B 12 (1998) 81.

[3] A. Avella, F. Mancini, V. Turkowski, Bosonic sector of the two-dimensional hubbard model studied within a two-pole approximation, Phys. Rev. B 67 (2003) 115123. 